\title{
Actividad física y su asociación con factores de riesgo cardiovascular. Un estudio en adultos jóvenes
}

\author{
ANTONIO ARTEAGA ${ }^{1}$, PATRICIA BUSTOS ${ }^{2}$, RODRIGO SOTO ${ }^{1}$, \\ NICOLÁS VELASCO ${ }^{1}$, HUGO AMIGO ${ }^{2}$
}

\section{Physical activity and cardiovascular risk factors among Chilean young men and women}

Background: Physical activity (PA) has a protective role in cardiovascular diseases. Aim: To quantify PA in young adults and to correlate it with cardiovascular risk factors. Material and Methods: A cross-sectional study was performed employing the international physical activity questionnaire (IPAQ), to measure the PA of 983 randomly selected young adults from Valparaiso region born between 1974 and 1978. Its results were associated with levels of obesity, insulin resistance and cardiovascular risk factors defined by the National Cholesterol Education Program-Adult Treatment Panel III (NCEP-ATP III) Results: Mean physical activity among men and women was $3731 \pm 3923$ and $1360 \pm 2303$ METs-minutes/week, respectively $(p<0.001)$. Fifty percent of women and $21.5 \%$ of men had an insufficient level of physical activity $(p<0.001)$. Sixty percent of men and $23.4 \%$ of women had an intense level of physical activity $(p<0.001)$. There was an inverse association of physical activity and insulin resistance. A high physical activity was protective, specially among men, against a low HDL cholesterol level and high triglyceride levels with Odds Ratios of 0.59 (confidence interval (CI): 0,35-0.98) and 0.49 (CI: 0,27-0,87) respectively, after adjusting for body mass index and age. Conclusions: In this sample, men had higher levels of physical activity, that was protective against insulin resistance and the presence of cardiovascular risk factors.

(Rev Med Chile 2010; 138: 1209-1216).

Key words: Cardiovascular diseases; Insulin resistance; Motor activity.

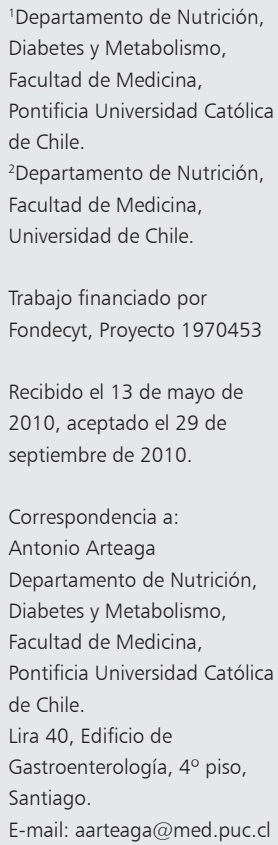

L as enfermedades cardiovasculares constituyen la primera causa de muerte en Chile ${ }^{1}$. El que en nuestro país exista además una elevada prevalencia de factores de riesgo (FR) cardiovascular y de sus condicionantes (obesidad y sedentarismo), permiten predecir un incremento de las tasas de enfermedades cardiovasculares ${ }^{2-5}$. Generalmente se acepta que el sedentarismo produce un incremento de estas enfermedades, especialmente infarto del miocardio y muerte súbita. Lo anterior ha llevado a incorporar la actividad física (AF) como herramienta de manejo clínico y preventivo para los FR y la enfermedad cardiovascular.

Las evidencias que permiten asociar AF y riesgo cardiovascular no son concluyentes debido a diferencias en la selección de la población, en los indicadores de AF utilizados, en la clasificación de los resultados y criterios para fijar objetivos terminales ${ }^{6-8}$. A pesar de lo anterior, la mayoría de los expertos ha considerado válido recomendar la $\mathrm{AF}^{9}$. Se han propuesto una serie de mecanismos biológicos para explicar cómo la AF puede reducir la morbi-mortalidad cardiovascular y corregir los 
FR cardiovasculares indicándose que el ejercicio podría reducir la resistencia insulínica (RI) y síndrome metabólico $(\mathrm{SM})^{10}$.

Existen distintos métodos para evaluar AF: objetivos y subjetivos. Entre los objetivos se describen sensores de movimientos, los podómetros, acelerómetros, y monitores de frecuencia cardíaca. Las técnicas subjetivas utilizan cuestionarios de AF, ya sea por auto-reporte o asistidos por entrevistador $^{11}$. Estos cuestionarios constituyen una herramienta de bajo costo y fácilmente aplicable a poblaciones, pero tienen una gran variabilidad. En vista de lo anterior, un grupo de expertos desarrollaron un método de evaluación de AF para ser utilizado a nivel poblacional: el International Physical Activity Questionnaire (IPAQ), el que fue sometido a un examen de confiabilidad y validez en 14 centros de 12 países, de lo que se concluyó que el instrumento provee mediciones aceptables de $\mathrm{AF}$ y sus resultados tienen una elevada correlación con los del acelerómetro ${ }^{12}$.

Los estudios desarrollados en nuestro país ${ }^{2-5}$ han destacado la alta prevalencia de sedentarismo, que bordea $90 \%$. Salvo la excepción de Jadue y $\mathrm{col}^{5}$, en estos trabajos no se han utilizado herramientas internacionalmente validadas para evaluar la AF. El objetivo de este trabajo fue evaluar la AF en una población de adultos jóvenes chilenos, por medio de un método validado internacionalmente, y estimar la relación existente entre AF y los FR cardiovascular.

\section{Material y Métodos}

Se estudió una población de adultos jóvenes de 22 a 28 años de ambos sexos, de la comuna de Limache, Región de Valparaiso. Cohorte reclutada en una investigación previa, seleccionada aleatoriamente de los nacidos entre 1974 y 1978 para estudiar la asociación entre características del recién nacido con la presencia de FR cardiovascular posteriores.

Como criterio de inclusión se consideró la capacidad de entender y contestar los datos de la encuesta. Se excluyeron embarazadas y nodrizas, y aquellas personas portadoras de enfermedades metabólicas o genéticas. De los 1.000 sujetos evaluados se analizaron 983 (427 hombres y 556 mujeres) excluyéndose aquellos con cuestionarios incompletos o información inconsistente. Este estudio fue aprobado por el comité de ética de la Facultad de Medicina de la Universidad de Chile y las personas participaron después de leer y firmar un consentimiento informado.

El estudio se efectuó en el hospital de Limache, en el consultorio de Olmué y en las postas rurales de la localidad durante los años 2000 a 2003. Se aplicó una encuesta para recoger datos demográficos, se evaluó la presión arterial (PA), la circunferencia de cintura, el peso y la estatura (con los que se calculo el índice de masa corporal (IMC)). Además se tomó una muestra de sangre, previo ayuno de 12 horas. La PA y las medidas antropométricas se midieron en condiciones estandarizadas por 3 enfermeras universitarias con entrenamiento en acuerdo a normas internacionales ${ }^{13}$. La circunferencia abdominal (pCC) se determinó con una cinta métrica flexible en el punto medio entre el reborde costal y cresta ilíaca considerandose un valor elevado sobre $88 \mathrm{~cm}$ en mujeres y $102 \mathrm{~cm}$ en hombres. La PA fue tomada 2 veces con un esfigmomanómetro (OMRON 740C) utilizándose en los análisis la media de las dos lecturas ${ }^{14}$. Se consideró como hipertenso a aquellos con presión sistólica (PAS) $\geq 140 \mathrm{o}$ diastólica (PAD) $\geq$ a $90 \mathrm{~mm} \mathrm{Hg}$ o si estaban con terapia hipotensora. En la medición del peso se utilizó una balanza con precisión de 100 g y en la estatura un cartabón con precisión de $1 \mathrm{~mm}$. Estas mediciones se realizaron sin zapatos y con ropa liviana. Los puntos de corte para definir sobrepeso fueron IMC entre 25 y 29,9 y obesidad $\geq 30$.

La muestra de sangre fue procesada y congelada en el hospital de Limache para su posterior análisis en el laboratorio de la Pontificia Universidad Católica de Chile. Se determinaron glicemia e insulinemia plasmática y se estimó el valor de HOMA. La glicemia se midió con método enzimático (GOD/PAP method, Human diagnostica, Alemania). La insulinemia se evaluó por RIA (Insulin Kit DPC. Los Angeles, EEUU). Para el cálculo de HOMA se utilizó la fórmula publicada por Matheus y cols ${ }^{15}, \mathrm{HOMA}=$ insulinemia $\mu \mathrm{U} /$ $\mathrm{ml} \mathrm{x}$ glicemia $\mathrm{mmol} / \mathrm{L} / 22,5$. Se consideró glicemia elevada cuando resulto ser $\geq 100 \mathrm{mg} / \mathrm{dL}$, resistencia a la insulina (RI) cuando era $\geq 2,53^{16}$, el colesterol total (C-total) se midió mediante método enzimático colorimétrico (Gesellschaft für Biochemica und Diagnostica mbh, Alemania), el colesterol HDL (C-HDL) por precipitación (Siegler y Wu) y los triglicéridos (TGS) por método enzimático 
(Gesellschaft für Biochemical und Diagnostica mbh, Alemania). Se consideró C-Total elevado $\geq$ $200 \mathrm{mg} / \mathrm{dl}$, TGS elevados $\geq 150 \mathrm{mg} / \mathrm{dL}$ y C-HDL bajos $<40 \mathrm{mg} / \mathrm{dL}$ para hombres $\mathrm{y}<50 \mathrm{mg} / \mathrm{dL}$ para mujeres ${ }^{17}$. El diagnóstico de SM se realizó según criterio del NCEP ATP III, versión modificada $2004^{17}$

Para la evaluación de la AF, se aplicó la encuesta IPAQ por personal previamente capacitado. Se usó la versión corta de esta encuesta, expresándola en METs-min/semana. Esta versión es un instrumento principalmente diseñado para estudios epidemiológicos en adultos (15-69 años). El IPAQ mide la AF realizada a través de una evaluación de 3 aspectos: AF intensa, AF moderada y caminar. El puntaje final requiere de la suma de duración (en minutos) y frecuencia (días) de las distintas actividades. La actividad física se analizó en forma continua y categorizada en actividad insuficiente (<600 METs min/semana), moderada (600-1.500 METs min/semana) e intensa (> 1.500 METs min/ semana $)^{18}$, controlando el efecto de factores de confusión e interacción.

Se verificó la consistencia de los datos. Se analizó la distribución de frecuencia, comparando medias y proporciones. Se construyeron modelos de regresión lineal para observar la asociación entre AF (medida en forma continua) con la presión y las variables metabólicas medidas también en forma contínua y posteriorente la AF categorizada en insuficiente, moderada e intensa con los factores de riesgo categorizados según puntos de corte sugeridos por NCEP-ATP III, controlando el efecto de variables de confusión e interacción.

\section{Resultados}

La edad promedio fue $24,8 \pm 1,6$ años $(24,9 \pm$ 6 en hombres y 24,7 $\pm 1,6$ en mujeres). Se pudo apreciar que los valores promedios de las variables antropométricas y metabólicas están en rangos normales salvo el IMC cuyo promedio se ubica en rango de sobrepeso. Con excepción de los niveles de C-total y HOMA, en todos los indicadores restantes hay diferencias entre ambos sexos, observándose valores significativamente mayores de PA, glicemia, y TGS bajo en hombres mientras que HDL fue mayor en mujeres (Tabla 1).

Los hombres realizaron significativamente mayor AF que las mujeres, los que se aprecia al analizar la variable en forma continua (Tabla 2a). $\mathrm{Al}$ analizar la $\mathrm{AF}$ según categoría de intensidad, se observó que $60 \%$ de los hombres realizaban $\mathrm{AF}$ intensa. A la inversa, 50\% de las mujeres tenían AF insuficiente, compatible con la calificación de sedentarias. Al analizar la cantidad de METs efectuados en cada categoría de actividad, no se observaron diferencias entre sexos en el nivel bajo y moderado, en cambio en el grupo de actividad alta el promedio de METs efectuados fue mayor en hombres (Tabla 2b).

En Tabla 3 se presenta la prevalencia de FR

Tabla 1. Variables antropométricas, metabólicas y presión arterial en adultos jóvenes

\begin{tabular}{|c|c|c|c|c|}
\hline & $\begin{array}{l}\text { Total }(n=983) \\
(x \pm D S)\end{array}$ & $\begin{array}{c}\text { Hombres }(n=427) \\
(x \pm D S)\end{array}$ & $\begin{array}{l}\text { Mujeres ( }(n=556) \\
(x \pm D S)\end{array}$ & $\mathbf{p}$ \\
\hline IMC (Kg/m²) & $25,8 \pm 4,5$ & $25,3 \pm 3,7$ & $26,2 \pm 5,0$ & 0,003 \\
\hline PAS (mmHg) & $114,6 \pm 13,5$ & $123,3 \pm 11,6$ & $107,9 \pm 10,8$ & $<0,0001$ \\
\hline PAD $(\mathrm{mmHg})$ & $72,5 \pm 8,8$ & $75,6 \pm 8,5$ & $70,1 \pm 8,2$ & $<0,0001$ \\
\hline Glicemia (mg/dl) & $85,9 \pm 9,9$ & $88,0 \pm 10,3$ & $84,2 \pm 9,1$ & $<0,0001$ \\
\hline C-Total (mg/dl) & $178,5 \pm 38,5$ & $176,2 \pm 36,4$ & $180,3 \pm 40,0$ & $<0,098$ \\
\hline $\mathrm{C}-\mathrm{HDL}(\mathrm{mg} / \mathrm{dl})$ & $41,4 \pm 11,7$ & $39,5 \pm 11,1$ & $42,8 \pm 12,0$ & $<0,0001$ \\
\hline Triglicéridos (mg/dl) & $112,5 \pm 70,0$ & $126,1 \pm 82,5$ & $102,0 \pm 56,0$ & $<0,0001$ \\
\hline HOMA & $2,5 \pm 1,6$ & $2,6 \pm 1,9$ & $2,5 \pm 1,4$ & 0,259 \\
\hline C. cintura $(\mathrm{cm})$ & $84,0 \pm 11,4$ & $85,2 \pm 9,7$ & $83,0 \pm 12,4$ & 0,003 \\
\hline
\end{tabular}

* Promedio y Desviación estándar. 
cardiovascular en la población total y por sexo. Destaca la elevada prevalencia de sobrepeso, obesidad, FR cardiovascular y SM en la población general. No se observaron diferencias según sexo para la glicemia de ayuno alterada, el C-total elevado, HOMA elevado y la presencia de SM. Los hombres tuvieron mayor prevalencia de HTA y TGS elevados. Las mujeres mayor prevalencia de obesidad, CC aumentada y C-HDL bajo.

$\mathrm{Al}$ analizar la asociación entre AF como variable continua con los indicadores de riesgo cardiovascular se encontró una asociación directa con PAS y PAD, e inversa para HOMA en población total y ésta última también en hombres (Tabla 4). Para estimar la razón de chance (OR), entre AF y los FR cardiovascular, se comparó el nivel moderado e intenso con el nivel de actividad insuficiente. La actividad moderada fue factor protector de C-total elevado, la AF intensa en tanto fue protector para HDL bajos, TGS y colesterol total aumentados.

Tabla 2. Actividad física por sexo en la población estudiada

\begin{tabular}{|c|c|c|c|c|}
\hline \multicolumn{5}{|c|}{ a) Expresado en forma continua } \\
\hline & Total & Hombres & Mujeres & $\mathbf{p}$ \\
\hline \multicolumn{5}{|c|}{ 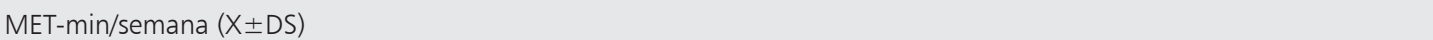 } \\
\hline & $2.389 \pm 3.324$ & $3.731 \pm 3.923$ & $1.360 \pm 2.303$ & $<0,001$ \\
\hline \multicolumn{5}{|c|}{ b) Expresado en categorías (\%, Nº de casos y promedios de MET-min/semana) } \\
\hline Nivel insuficiente & $\begin{array}{c}37,6 \quad(370) \\
223 \pm 182 \\
\text { Mets-min/sem }\end{array}$ & $\begin{array}{c}21,5 \quad(92) \\
248 \pm 202 \\
\text { Mets-min/sem }\end{array}$ & $\begin{array}{c}50,0 \quad(278) \\
214 \pm 174 \\
\text { Mets-min/sem }\end{array}$ & $\begin{array}{l}<0,001^{*} \\
\mathrm{NS}^{* *}\end{array}$ \\
\hline Nivel moderado & $\begin{array}{c}23,0 \quad(226) \\
1.001 \pm 262 \\
\text { Mets-min/sem }\end{array}$ & $\begin{array}{c}18,3 \quad(78) \\
1.023 \pm 250 \\
\text { Mets-min/sem }\end{array}$ & $\begin{array}{c}26,6 \quad(148) \\
988 \pm 267 \\
\text { Mets-min/sem }\end{array}$ & $\begin{array}{l}<0,001 * \\
\mathrm{NS}^{* *}\end{array}$ \\
\hline Nivel intenso & $\begin{array}{c}39,4 \quad(387) \\
5.272 \pm 3.753 \\
\text { Mets-min/sem }\end{array}$ & $\begin{array}{c}60,2 \quad(257) \\
5.719 \pm 3.822 \\
\text { Mets-min/sem }\end{array}$ & $\begin{array}{c}23,4 \quad(130) \\
4.230 \pm 2.372 \\
\text { Mets-min/sem }\end{array}$ & $\begin{array}{l}<0,001^{*} \\
<0,001^{* *}\end{array}$ \\
\hline
\end{tabular}

Nivel Insuficiente: < 600 MET-minuto semana. Nivel Moderado: 600-1.499 MET-minutos semana. Nivel Intenso: $\geq 1.500$ METminuto/semana. * diferencias entre porcentajes; ${ }^{* *}$ diferencias entre MET-min/semana.

Tabla 3. Prevalencia de factores de riesgo cardiovascular en población total y por sexo

\begin{tabular}{|c|c|c|c|c|}
\hline Condición & $\begin{array}{l}\text { Total } \\
\% \text { (n) }\end{array}$ & $\begin{array}{c}\text { Hombres } \\
\% \text { (n) }\end{array}$ & $\begin{array}{l}\text { Mujeres } \\
\% \text { (n) }\end{array}$ & $\mathbf{p}$ \\
\hline Sobrepeso & 34,5 (339) & $37,9(162)$ & $31,8(177)$ & 0,050 \\
\hline Obesidad & $15,8(155)$ & $10,5 \quad(45)$ & $19,8(110)$ & $<0,001$ \\
\hline CC aumentada & $19,6(193)$ & $4,9 \quad(21)$ & $30,9(172)$ & $<0,001$ \\
\hline Hipertensión & $5,9 \quad(58)$ & $10,8 \quad(46)$ & $2,2 \quad(12)$ & $<0,001$ \\
\hline Glicemia alterada & $5,3 \quad(52)$ & $6,6 \quad(28)$ & $4,3 \quad(24)$ & 0,12 \\
\hline C-Total elevado & $26,2(258)$ & 25,8 (110) & 26,6 (148) & 0,78 \\
\hline TGS elevados & 17,7 (174) & $23,0 \quad(98)$ & $13,6 \quad(76)$ & $<0,001$ \\
\hline C-HDL bajo & $66,8 \quad(657)$ & $55,3 \quad(236)$ & $75,7 \quad(421)$ & $<0,001$ \\
\hline HOMA elevado & $34,7 \quad(341)$ & $36,1 \quad(154)$ & $33,6(187)$ & 0,420 \\
\hline S. Metabólico & 11,8 (116) & $11,5 \quad(54)$ & $12,0 \quad(67)$ & 0,790 \\
\hline
\end{tabular}


Actividad física y riesgo cardiovascular - A. Arteaga et al

Tabla 4. Asociación entre actividad física y factores de riesgo metabólicos, ajustado por edad e IMC. Población total y por sexo

\begin{tabular}{|c|c|c|c|c|c|c|c|}
\hline & PAS & PAD & Glicemia & C- Total & TGS & C-HDL & HOMA \\
\hline \multicolumn{8}{|l|}{ Total } \\
\hline Coef B* & 0,783 & 0,195 & 0,097 & $-0,685$ & 0,575 & 0,069 & $-0,045$ \\
\hline IC & $0,5-1,0$ & $0,03-0,4$ & $-0,09-0,3$ & $-1,4-0,04$ & $-0,9-1,7$ & $-0,2-0,3$ & $-0,1--0,02$ \\
\hline$p=$ & $<0,001$ & 0,021 & 0,304 & 0,065 & 0,575 & 0,541 & 0,003 \\
\hline \multicolumn{8}{|c|}{ Hombres } \\
\hline Coef $B^{*}$ & 0,04 & $-0,078$ & 0,200 & $-0,670$ & $-0,839$ & 0,234 & $-0,070$ \\
\hline IC & $-0,2-0,3$ & $-0,3-0,13$ & $-0,5-0,05$ & $-1,6-0,2$ & $-2,8-1,2$ & $-0,03-0,5$ & $-0,1--0,03$ \\
\hline$p=$ & 0,765 & 0,460 & 0,115 & 0,139 & 0,411 & 0,090 & 0,002 \\
\hline \multicolumn{8}{|l|}{ Mujeres } \\
\hline Coef B* & $-0,202$ & $-0,196$ & 0,056 & $-0,233$ & $-1,41$ & 0,373 & $-0,033$ \\
\hline IC & $-0,6-0,19$ & $-0,5-0,1$ & $-0,3-0,4$ & $-1,7-1, .2$ & $-3,4-0,6$ & $-0,06-0,8$ & $-0,08-0,02$ \\
\hline$p=$ & 0,307 & 0,193 & 0,740 & 0,753 & 0,167 & 0,092 & 0,180 \\
\hline
\end{tabular}

*Coeficiente B cada 1000 MET.

\begin{tabular}{|c|c|c|c|c|c|c|}
\hline \multicolumn{2}{|c|}{ HOMBRES } & $\begin{array}{l}\text { Mediana/ } \\
\text { Insuficiente }\end{array}$ & & $\begin{array}{c}\text { Intensa/ } \\
\text { insuficiente }\end{array}$ & & $\begin{array}{l}\text { Intensa/ } \\
\text { insuficiente } \\
\text { corregida }\end{array}$ \\
\hline & OR & & OR & & OR & \\
\hline HDL & 0,99 & ג & 0,55 & مـ: * & 0,59 & * \\
\hline TGS & 0,73 & 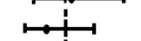 & 0,43 & คr * & 0,49 & $\mapsto$ * \\
\hline C-TOT & 0,88 & * & 0,59 & $\mapsto$ * & 0,49 & $\mapsto$ \\
\hline GLI & 2,53 & & 2,23 & $\mapsto$ & 2,33 & $\vdash$ \\
\hline HTA & 0,82 & $\mapsto$ & 0,62 & $\mapsto$ & 064 & $\rightarrow$ \\
\hline pCC & 0,42 & $\mapsto$ & 0,43 & $\mapsto \quad$ & & \\
\hline \multicolumn{7}{|c|}{ MUJERES } \\
\hline & OR & & OR & & OR & \\
\hline HDL & 0,97 & $\longmapsto$ & 1,25 & $\omega_{H}^{+1}$ & 1,18 & \\
\hline TGS & 1,88 & * & 1,72 & $\mapsto$ & 1,52 & \\
\hline C-TOT & 1,40 & $\mapsto$ & 1,28 & $\mapsto$ & 1,21 & - \\
\hline $\begin{array}{l}\text { GLI } \\
\text { HTA }\end{array}$ & 1,40 & $\mapsto$ & 1,01 & $\longmapsto$ & 0,85 & 1 \\
\hline pCC & $\begin{array}{l}1,00 \\
0,92\end{array}$ & 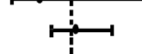 & $\begin{array}{l}1,65 \\
169\end{array}$ & $\mapsto$ & 1,33 & \\
\hline \multicolumn{7}{|c|}{$p=<0,05$} \\
\hline
\end{tabular}

Figura 1. Asociación entre actividad física y factores de riesgo cardiovasculares.
$\mathrm{Al}$ ajustar por IMC y edad la protección de la AF intensa persiste para TGS altos y C-HDL bajo. (Figura 1). En la mujer, al comparar el nivel moderado con insuficiente, sólo se aprecia una reducción del riesgo de presentar TGS altos y al comparar AF intensa con insuficiente el ejercicio intenso es un factor protector de CC elevada. Al ajustar por IMC y edad desaparecen estas asociaciones. Se apreció una asociación lineal inversa entre AF e IMC $(B=-0103 p=0,016)$. En la Tabla 5 observamos la asociación directa entre IMC y los FR cardiovasculares en población total y por sexo, observándose 
Tabla 5. Asociación entre IMC y variables en estudio. Población total y por sexo

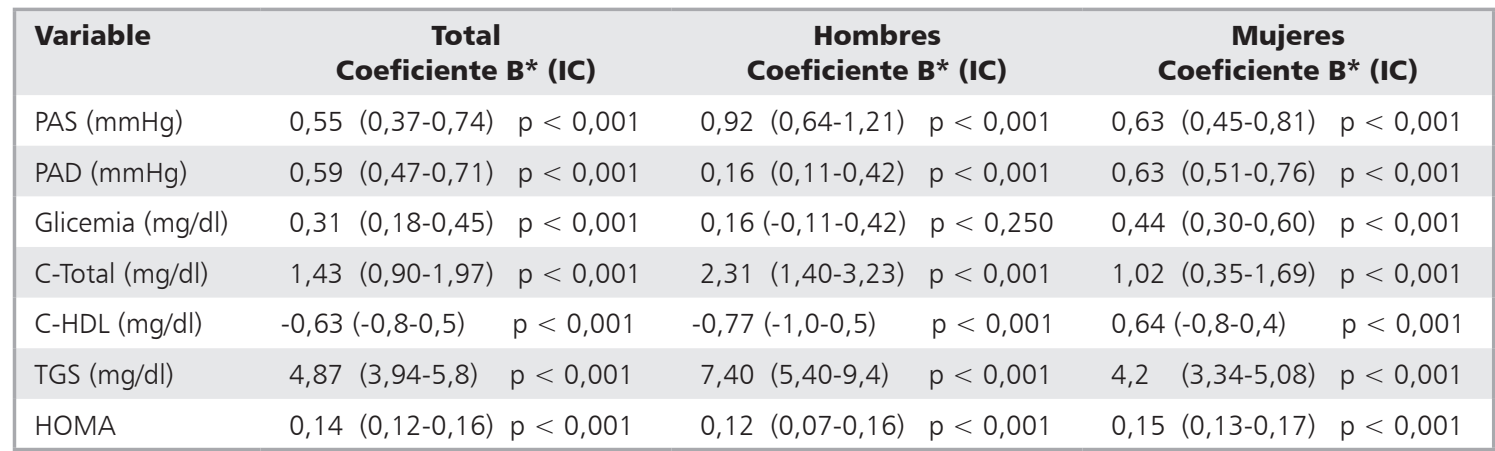

* Variación de variable en estudio por cada 1000 MET-min/semana.

que a medida que incrementa el IMC, aumentan los factores de riesgo con excepción de la glicemia en ayunas en hombres.

\section{Discusión}

La actividad física de esta población demuestra un comportamiento diferenciado entre mujeres y hombres: mientras la mitad de las mujeres tienen actividad física insuficiente, $60 \%$ de los hombres tienen actividad física intensa. $\mathrm{Al}$ analizar la variable AF en forma continua se observó un efecto inverso de ésta, con HOMA sólo en hombres. En relación a la asociación entre actividad física y factores de riesgo cardiovascular, hay un efecto protector de la actividad intensa -sólo en hombres- para no tener C-HDL bajos y triglicéridos aumentados.

Existen controversias acerca del tiempo e intensidad de la $\mathrm{AF}$ requerida para reducir los FR cardiovascular. Entre las explicaciones estarían las diferencias de diseño de los estudios y las dificultades técnicas para evaluar la AF a nivel poblacional. Nuestro estudio se realizó en una población joven de un área agro-industrial, en la cual era previsible encontrar una amplia distribución de los niveles de AF, a diferencia de zonas urbanas en donde predomina el sedentarismo. Para estimar AF, seleccionamos una metodología de encuesta validada que permite analizar la información como una variable continua y categórica. Los criterios de categorización de IPAQ son consensuados y de alta aceptación internacional ${ }^{12}$. La categoría intermedia es similar a las recomendaciones estándar de AF de la American Heart Association ${ }^{9}$, y la actividad insuficiente puede homologarse con el sedentarismo. En el IPAQ se reconoce que los puntos de corte son arbitrarios y pudieran ser reconsiderados, por lo que exploramos el análisis de la efectividad de la AF además como variable continua. En el país hay pocas encuestas de AF a nivel poblacional que permitan realizar comparaciones y las existentes han sido hechas con distintas metodologías.

En el presente estudio, 50\% de la mujeres y $21,5 \%$ de los hombres corresponden a la categoría definida como sedentaria, porcentajes menores a lo observado en la Encuesta Nacional de Salud que fue cercano a $90 \%{ }^{19}$. Las comparaciones con otros estudios nacionales resultan difíciles, ya que en ellos la AF fue estimada por métodos diferentes. En Valparaíso, Chile, Jadue y col utilizaron el formulario de Baecke en un grupo de edad semejante al nuestro (20-30 años). La prevalencia de AF media y alta combinada fue cercana a $20 \%$, inferior a lo reportado en nuestro estudio ${ }^{5}$. Esta diferencia podría ser explicada por características poblacionales, ya que la población de Valparaíso es predominantemente urbana, en cambio la nuestra fue rural agro-industrial.

Una de las limitaciones de nuestro estudio es la evaluación subjetiva de la AF. Sin embargo, se debe destacar la validez internacional del IPAQ, lo que permite confiar en la reproducibilidad de los datos obtenidos. A pesar de que nuestra población era joven, nuestro estudio reveló una elevada prevalencia de FR y diferencias entre ambos sexos, lo que ya se había comunicado en una descripción preliminar de esta población ${ }^{20}$. Un hecho destacado, fue el diferente impacto de la AF por sexo 
sobre los FR. En efecto en la mujer no se apreció una asociación con la resistencia insulínica, ni un efecto independiente sobre los otros factores de riesgo. Es difícil explicar esta divergencia. Es posible especular que se debiera a diferencias en la intensidad promedio de la AF por categoría, lo cual se observa en la categoría de mayor intensidad, en la que los hombres realizaron una AF promedio mayor que las mujeres. Sin embargo, no es posible descartar que se deban a diferencias estructurales y fisiológicas relacionadas al sexo.

$\mathrm{Al}$ analizar la relación de la AF y el HOMA como variables continuas, observamos sólo en los hombres una asociación positiva independiente, lo que apoya el rol de la AF en el tratamiento y prevención de la resistencia insulínica en este grupo. Existe cierta discrepancia acerca de este efecto, sin embargo, en observaciones controladas, ello ha sido observado y atribuido a un efecto independiente de la actividad muscular sobre la sensibilidad insulínica ${ }^{10}$. Posiblemente esta discordancia dependa del diseño de esos estudios, en los que la AF fue categorizada como sedentaria y no sedentaria, reduciendo el peso de la actividad mayor sobre la sensibilidad a la insulina. También en el hombre, la AF intensa se asoció con una menor frecuencia de hipercolesterolemia, hipertrigliceridemia, C-HDL bajo, siendo sólo el efecto sobre el C-HDL y triglicéridos, independiente del IMC.

Resulta difícil explicar la asociación directa observada entre la AF y niveles de PA, es posible que ello pudiera reflejar una disociación entre capacidad aeróbica e intensidad de la AF, en este grupo de población.

Nuestro trabajo muestra una relación entre $\mathrm{AF}$ e IMC. En nuestro caso, la AF presentó una asociación inversa con el IMC, y este último se asoció a todos los factores estudiados. Ello sugiere que el efecto de la AF se ejerce en parte modulando el peso corporal, lo que puede ayudar a entender discordancias acerca de la influencia de la AF sobre los factores de riesgo, pues su medición puede reflejar un hábito reciente y en cambio el IMC es el resultado a mediano y largo plazo de una serie de factores, entre los que se encuentra la propia actividad física.

Este estudio apoya el rol protector de la AF en el hombre sobre la resistencia insulínica y algunos FR estudiados lo que se hace aparente a niveles mayores (más de $1.500 \mathrm{METs}$-minutos/semana). Esto sugiere replantear las recomendaciones vi- gentes $^{9}$, para aquellos que la puedan desarrollar sin riesgos adicionales.

En conclusión, la AF parece ser un factor protector del riesgo cardiovascular, hecho que es importante cuando esta se efectúa a mayor intensidad. La diferencia de impacto en ambos sexos, deberá ser corroborada en otros estudios y analizadas sus consecuencias. El ejercicio físico vigoroso y programado debiera ser considerado como parte de las estrategias primordiales de prevención cardiovascular en la población de adultos jóvenes, en especial en aquellos involucrados en ocupaciones con AF poco intensa.

\section{Referencias}

1. Instituto Nacional de Estadísticas [homepage on the Internet]. Chile: Instituto Nacional de Estadísticas; c2003 [citado 20 Feb 2010]. [aprox. 3 pantallas]. Disponible en: http://www.ine.cl/canales/chile_estadistico/ demografia_y_vitales/estadisticas_vitales/pdf/causas_ de_muerte_regiones\%202003.PDF

2. Vio DF. Prevención de la Obesidad en Chile. Rev Chil Nutr 2005; 32: 80-7.

3. Berríos X, Jadue L, Zenteno J, Ross M, Rodríguez H. Prevalencia de Factores de Riesgo de Enfermedades Crónicas. Estudio en Población General de la Región Metropolitana, 1986-1987. Rev Med Chile 1990; 118: 597-604.

4. Berríos X. Tendencia Temporal de los Factores de Riesgo de Enfermedades Crónicas: ¿La Antesala Silenciosa de una Epidemia que Viene? Rev Med Chile 1997; 125: 1405-7.

5. Jadue L, Vega J, Escobar M, Delgado I, Garrido C, Lastra $\mathrm{P}$, et al. Factores de Riesgo para las enfermedades no transmisibles: Metodología y Resultados Globales de la Encuesta de Base del Programa CARMEN (Conjunto de Acciones para la Reducción Multifactorial de las Enfermedades no Transmisibles). Rev Med Chile 1999; 127: 1004-13.

6. Berlin JA, Golditz GA. A meta-analysis of physical activity in the prevención of coronary heart disease. American J Epidemiol 1990; 132: 612-28.

7. Powell KE, Thompson PD, Caspersen CJ, Kendrech JS. Physical activity and incidence of coronary artery disease. Ann Rev Public Health 1987; 8: 253-87.

8. Cassel J, Heyden S, Bartel AG, Kaplan BH, Tyroler HA, Cornoni JC, et al. Occupation and physical activity and coronary heart disease Arch Int Med 1971; 128: 920-6.

9. Haskell WL, Lee IM, Pate RR, Powell KE, Blair SN, Franklin BA, et al. Physical activity and public health: 
updated recommendation for adults from the American College of Sports Medicine and the American Heart Association. Circulation 2007; 116: 1081-93.

10. Henriksson J. Influence of excercise on insulin sensibility. J Cardiovascular Risk 1995; 2: 303-8.

11. Vanhees L, Lefevre J, Philippaerts R, Martens M, Huygens $\mathrm{W}$, Troosters $\mathrm{T}$, et al. How to assess physical activity? How to assess physical fitness? Eur J Cardiovasc Prev Rehabil 2005; 12: 102-14.

12. Craig C, Marshall A, Sjöström M, Barman A, Booth M, Ainsworth B, et al. International Physical Activity Questionnaire: 12-Country Reliability and Validity. Med Sci Sports Exerc 2003; 35: 1381-95.

13. Habitch JP. Estandarización de métodos epidemiológicos cuantitativos sobre el terreno. Bol of Sanit Panam 1974; 76: 375-84.

14. O'Brien E. Evaluation of three devices for sel measurement of blood pressure according to the revised British Hipertension Society Protocol: The Omron HEM-705 CP, Philips HP 5332 and Nissei DS-175. Blood Press Monit 1996; 1: 55-61.

15. Mattheus DR, Hosker JP, Rudenski AS, Naylor BA, Treacher DF, Turner RC. Homeostasis Model Assesment: Insulin resistance and B-cell function from fasting plasma glucose and insulin concentrations in man. Diabetologia 1985; 28: 412-9.

16. Acosta AM. [Determination of Insulin Resistance index by the Homeostasis Model Assesment in a Population of Metropolitan Region in Chile]. Rev Med Chile 2002; 130 (11): 1227-31.

17. National Heart, Lung and Blood Institute, National Institutes of Health [homepage on the Internet]. Estados Unidos: Expert Panel on Detection, Evaluation and Treatment of High Blood Cholesterol in Adults; c2002 [actualizdo 2004; citado 20 Feb 2010]. [aprox. 2 pantallas]. Disponible en: http://www.nhlbi.nih.gov/ guidelines/cholesterol/index.htm

18. IPAQ Research Committee [homepage on the Internet]. Suecia: IPAQ Research Committee; c2005 [citado $20 \mathrm{Feb}$ 2010]. [aprox. 2 pantallas]. Disponible en: http://www. ipaq.ki.se/ipaq.htm.

19. Ministerio de Salud [homepage on the Internet]. Chile: Departamento de Epidemiología Ministerio de Salud; c2004 [citado 20 Feb 2010]. [aprox. 2 pantallas]. Disponible en: http://epi.minsal.cl/epi/html/invest/ENS/ENS.htm

20. Bustos P, Amigo H, Arteaga A, Acosta AM, Rona R. Factores de riesgo de enfermedad cardiovascular en adultos jóvenes. Rev Med Chile 2003; 131: 973-80. 